\title{
Mutational signatures and chromosome alteration profiles of squamous cell carcinomas of the vulva
}

\author{
Mi-Ryung Han ${ }^{1,2}$, Sun Shin ${ }^{1,2,3}$, Hyeon-Chun Park ${ }^{1,2}$, Min Sung Kim ${ }^{4,5}$, Sung Hak Lee ${ }^{6}$, Seung Hyun Jung ${ }^{1,2,5}$, \\ Sang Yong Song ${ }^{7}$, Sug Hyung $\mathrm{Lee}^{4,5}$ and Yeun-Jun Chung ${ }^{1,2,3}$
}

Vulvar squamous cell carcinoma (SCC) consists of two different etiologic categories: human papilloma virus (HPV)-associated (HPV (+)) and HPV-non-associated (HPV (-)). There have been no genome-wide studies on the genetic alterations of vulvar SCCs or on the differences between HPV (+) and HPV (-) vulvar SCCs. In this study, we performed whole-exome sequencing and copy number profiling of $6 \mathrm{HPV}(+)$ and $9 \mathrm{HPV}(-)$ vulvar SCCs and found known mutations (TP53, CDKN2A and HRAS) and copy number alterations (CNAs) (7p and 8q gains and $2 q$ loss) in HPV (-) SCCs. In HPV (+), we found novel mutations in PIK3CA, BRCA2 and FBXW7 that had not been reported in vulvar SCCs. HPV $(-)$ SCCs exhibited more mutational loads (numbers of nonsilent mutations and driver mutations) than HPV (+) SCCs, but the CNA loads and mutation signatures between HPV (+) and HPV (-) SCCs did not differ. Of note, $40 \%$ and $40 \%$ of the 15 vulvar SCCs harbored PIK3CA and FAT1 alterations, respectively. In addition, we found that the SCCs harbored kataegis (a localized hypermutation) in 2 HPV (+) SCCs and copyneutral losses of heterozygosity in 4 (one HPV (+) and 3 HPV (-)) SCCs. Our data indicate that HPV (+) and HPV (-) vulvar SCCs may have different mutation and CNA profiles but that there are genomic features common to SCCs. Our data provide useful information for both HPV (+) and HPV (-) vulvar SCCs and may aid in the development of clinical treatment strategies.

Experimental \& Molecular Medicine (2018) 50, e442; doi:10.1038/emm.2017.265; published online 9 February 2018

\section{INTRODUCTION}

Vulvar cancer is a malignant invasive lesion occurring in the vaginal opening, the labia majora (the most common site), the labia minora and the clitoris. Vulvar cancer accounts for $0.6 \%$ of all cancer diagnoses and $5 \%$ of gynecologic cancers. ${ }^{1,2}$ Vulvar cancer is typically a squamous cell carcinoma (SCC) that is also common in other gynecologic organs, including the cervix and vagina. ${ }^{1,2}$ Whereas almost all cervical SCCs occur with the background of human papillomavirus (HPV) infection, ${ }^{3,4}$ vulvar SCCs consist of those associated with HPV as well as others independent of HPV infection. ${ }^{1,2}$ Both HPV (+) and HPV (-) vulvar SCCs are preceded by vulvar intraepithelial neoplasia (VIN). ${ }^{5,6}$ These two types of vulvar SCCs along with their precursors exhibit different epidemiological, pathological, clinical and molecular features. ${ }^{1,2,5,6}$ Approximately one-third of all vulvar SCC patients suffer from recurrence, for which therapeutic options are limited. ${ }^{7}$

SCCs developed in female genital tracts share common features, including HPV infection and the progression of squamous intraepithelial neoplasia to invasive SCCs. ${ }^{3,4}$ Like cervical intraepithelial neoplasia, VINs progress to SCCs but the progression rates are lower than those of cervical lesions, ${ }^{5,6}$ indicating that the pathogenesis of cervical and vulvar SCCs may differ in part.

With the improvement of next-generation sequencing (NGS) technology, comprehensive molecular profiles of many cancers have been studied using NGS, ${ }^{8}$ which allows for the

\footnotetext{
${ }^{1}$ Department of Integrated Research Center for Genome Polymorphism, The Catholic University of Korea, Seoul, Korea; ${ }^{2}$ Precision Medicine Research Center, The Catholic University of Korea, Seoul, Korea; ${ }^{3}$ Department of Microbiology, The Catholic University of Korea, Seoul, Korea; ${ }^{4}$ Department of Pathology, The Catholic University of Korea, Seoul, Korea; ${ }^{5}$ Cancer Evolution Research Center, The Catholic University of Korea, Seoul, Korea; ${ }^{6}$ Department of Hospital Pathology, The Catholic University of Korea, Seoul, Korea and ${ }^{7}$ Department of Pathology and Translational Genomics, Samsung Medical Center, Sungkyunkwan University School of Medicine, Seoul, Korea

Correspondence: Professor SY Song, Department of Pathology and Translational Genomics, Samsung Medical Center, Sungkyunkwan University School of Medicine 81 IIwon-ro, Kangnam-gu, Seoul 06351, Korea.

E-mail: yodasong@hanmail.net

or Professor SH Lee, Department of Pathology, The Catholic University of Korea, Seoul, Korea.

or Professor YJ Chung, Precision Medicine Research Center, The Catholic University of Korea, College of Medicine, 222 Banpo-daero, Socho-gu, Seoul 06591, Korea.

E-mail: suhulee@catholic.ac.kr or yejun@catholic.ac.kr

Received 31 May 2017; revised 30 August 2017; accepted 4 September 2017
} 
investigation of thousands of variants within a given tumor sample. ${ }^{9-12}$ Using a targeted sequencing approach for 14 genes, a recent study identified that most HPV-negative (-) vulvar SCCs $(83 \%)$ contained one or more somatic mutations in TP53, CDKN2A, HRAS, KRAS, PIK3CA, PPP2R1A and PTEN, with TP53 being the most commonly mutated gene. ${ }^{13}$ In contrast, HPV-positive (+) vulvar SCCs harbored a TP53 mutation in $17 \%$ of cases but the remaining $83 \%$ were silent without any driver mutations. ${ }^{13}$ Their study strongly suggests not only that HPV-dependent and HPV-independent vulvar SCCs may have different mutation profiles but also there could be hidden mutations not discovered by the targeted approach only for the 14 genes. Other studies using conventional geneto-gene analyses have shown similar mutation data in vulvar SCCs. ${ }^{1,2,14,15}$ Thus far, a number of studies have reported the mutational profiles of SCC in many organs (non-genital: head/ neck, esophagus and skin; genital: uterine cervix and penis) using high-throughput genome profiling technologies. ${ }^{11,16-19}$ However, to date, the genomic data of vulvar SCCs at the whole-genome or whole-exome level is absent. Thus, it is necessary to examine the mutational profiles of vulvar SCCs in addition to the well-known gene mutations, including TP53. In this study, we analyzed the genomes of 15 vulvar SCCs using NGS-based whole-exome sequencing (WES) to identify the mutational profiles of vulvar SCCs.

\section{MATERIALS AND METHODS}

\section{Vulvar cancer tissues}

Vulvar SCC tissues resected by surgery were obtained from 15 Korean patients. Tissues from one patient were frozen (VSCC2) and those from the other 14 patients were formalin-fixed paraffin-embedded (FFPE). This study was approved by the Institutional Review Board (IRB) at The Catholic University of Korea, College of Medicine. Clinicopathologic characteristics of the 15 vulvar SCC patients are summarized in Table 1. Both frozen and FFPE tissues were cut and examined under a microscope by two pathologists. Tumor and normal cells were selectively procured from hematoxylin-stained sections by microdissection. The purity of the tumor cells from the microdissection was approximately $70 \%$. HPV (+) tumors were determined using real-time polymerase chain reaction (PCR) for HPV obtained from a commercial company for molecular diagnosis (Seegene, Inc., Seoul, Korea).

\section{WES and somatic mutations}

WES was performed on the genomic DNA obtained from tumor and matched normal tissues using the Agilent SureSelect Human All Exome V4 Kit (Agilent Technologies, Santa Clara, CA, USA) according to the manufacturer's instructions. WES data were processed as previously described. ${ }^{20}$ Briefly, the paired-end sequencing raw data (FASTQ format) were aligned to the human reference genome (hg19) using Burrows-Wheeler aligner software (BWA, v0.7.15). ${ }^{21}$ The Genome Analysis toolkit (GATK, v3.6), ${ }^{22}$ Picard (http://picard.sourceforge.net; v2.7.1) and Samtools $(v 1.3 .1)^{23}$ were used for the basic processing and management of marking duplicates, including local realignments and score recalibration. Somatic mutations were detected by comparing tumor and matched normal sequencing data using MuTect $^{24}$ and SomaticIndelDetector ${ }^{22}$ for point mutations and indels, respectively. ANNOVAR (Annotate Variation) was used for the functional annotation of each variant in the coding regions. ${ }^{25}$ PolyPhen-2 was used to predict the impact of an amino-acid substitution on protein function and structure. ${ }^{26}$ We validated 21 mutations of 9 genes using either digital PCR or Sanger sequencing. Putative regions of kataegis were identified in samples where mutations had an average inter-mutation distance of no more than $10 \mathrm{~kb} .^{27}$

\section{Comprehensive analysis of mutational signatures}

Somatic mutation signatures were estimated using the SomaticSignatures $\mathrm{R}$ package, ${ }^{28}$ in which a mutation spectrum was decomposed with a non-negative matrix factorization (NMF) algorithm. Mutational signature analyses were rerun 20 times to confirm the results

Table 1 Clinicopathologic features of vulvar cancers

\begin{tabular}{|c|c|c|c|c|c|c|}
\hline Sample & Age (years) & Tumor size in diameter $(\mathrm{cm})$ & Diagnosis & Extent of carcinoma & HPV infection (high- and low-risk types) & Sample source \\
\hline VSCC1 & 60 & 3.5 & Vulva SCC & Microinvasion & $+($ HPV16, - ) & FFPE \\
\hline VSCC2 & 74 & 3 & Vulva SCC & invasion (7.0 mm) & $+($ HPV16, - ) & Frozen \\
\hline VSCC3 & 78 & 3 & Vulva SCC & Invasion (8.0 mm) & $+($ HPV58, - ) & FFPE \\
\hline VSCC4 & 61 & 0.5 & Vulva SCC & Microinvasion & $+($ HPV16, - $)$ & FFPE \\
\hline VSCC5 & 66 & 2 & Vulva SCC & Invasion (6.0 mm) & $+($ HPV52, -$)$ & FFPE \\
\hline VSCC6 & 47 & 1.5 & Vulva SCC & Invasion (1.0 mm) & $+($ HPV16, - ) & FFPE \\
\hline VSCC7 & 70 & 2.5 & Vulva SCC & Invasion (7.0 mm) & - & FFPE \\
\hline VSCC8 & 70 & 4 & Vulva SCC & Invasion (4.0 mm) & - & FFPE \\
\hline VSCC9 & 45 & 1.3 & Vulva SCC (VIN) & No invasion & - & FFPE \\
\hline VSCC10 & 61 & 0.6 & Vulva SCC & Invasion (1.4 mm) & - & FFPE \\
\hline VSCC11 & 62 & 2.4 & Vulva SCC & Invasion (1.0 mm) & - & FFPE \\
\hline VSCC12 & 69 & 4 & Vulva SCC & Invasion (7.0 mm) & - & FFPE \\
\hline VSCC13 & 79 & 4 & Vulva SCC & Invasion (1.0 mm) & - & FFPE \\
\hline VSCC14 & 64 & 1.7 & Vulva (VIN) SCC & No invasion & - & FFPE \\
\hline VSCC15 & 83 & 1.2 & Vulva SCC & Invasion (4.0 mm) & - & FFPE \\
\hline
\end{tabular}

Abbreviations: FFPE, formalin-fixed paraffin-embedded; HPV, human papilloma virus; VIN, vulva intraepithelial neoplasm.

HPV infection risk types are indicated in parentheses (high risk, low risk).

High-risk HPVs are indicated by the numbers 16,52 , and 58. 
a

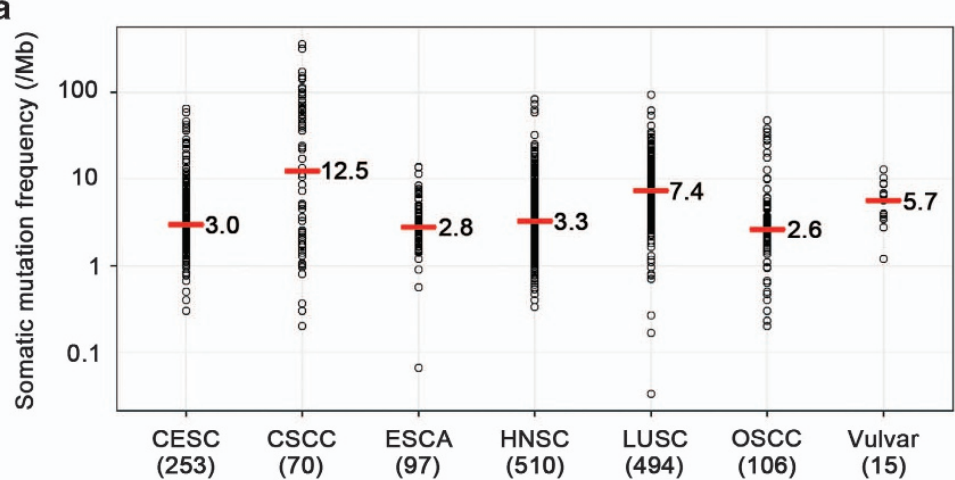

b
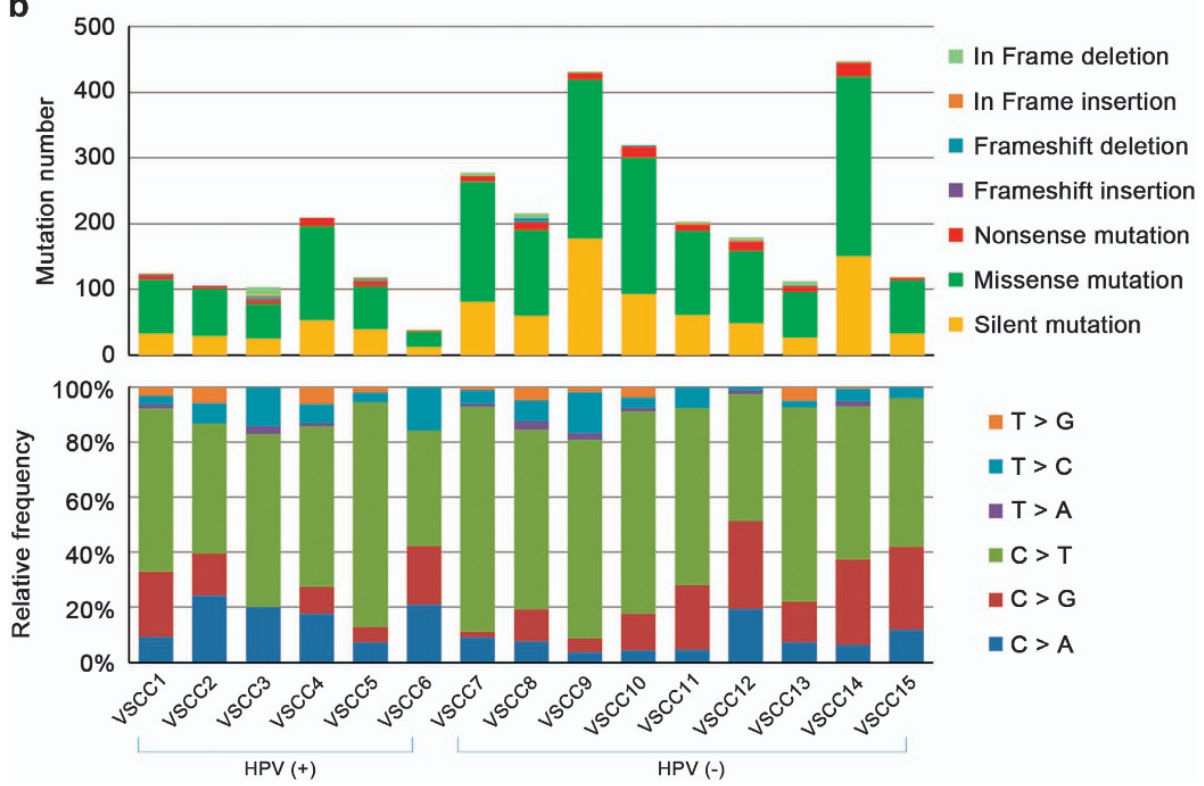

Figure 1 Compositions of somatic mutations identified in vulvar cancer genomes. (a) Mutation frequency in vulvar cancer genomes compared with those in other squamous cell carcinomas (CESC, cervical squamous cell carcinoma; CSCC, skin cutaneous squamous cell carcinoma; ESCA, esophageal squamous cell carcinoma; HNSC, head and neck squamous cell carcinoma; LUSC, lung squamous cell carcinoma; OSCC, oral squamous cell carcinoma; Mb, megabase). The median mutation frequency value is indicated next to each bar. Numbers of cases for each squamous cell carcinoma are shown in parentheses. (b) Number of somatic mutations (upper plot) and type of base pair substitution (lower plot) of 15 vulvar SCC genomes (6 HPV (+) and 9 HPV (-)) are shown. A total of 2084 nonsilent and 924 silent mutations were identified in 15 vulvar SCC genomes.

and an optimal number of signatures was chosen based on the maximum differentiation between the signatures. To compare the mutation signatures of the 15 vulvar SCCs with other cancer types, we first calculated the similarities between the mutational signatures using 30 known signatures recorded in the Catalogue of Somatic Mutations in Cancer (COSMIC) database (http://cancer.sanger.ac.uk/cosmic/ signatures). ${ }^{29}$ Similarities between mutational signatures were calculated using a cosine correlation similarity ranging from 0 and 1 , where one represented identical signatures and zero represented completely non-identical signatures. Next, we systematically analyzed the mutational signatures of SCCs using data from The Cancer Genome Atlas $(\text { TCGA })^{30}$ and a comprehensive literature review of NGS-based studies for SCCs. ${ }^{11,16,31-33}$ Data were composed of four types of SCCs from TCGA (CESC: cervical squamous cell carcinoma, ESCA: esophageal squamous cell carcinoma, HNSC: head and neck squamous cell carcinoma, LUSC: lung squamous cell carcinoma) $)^{30}$ and two SCCs from NGS-based studies (CSCC: skin cutaneous squamous cell carcinoma, ${ }^{11,16}$ OSCC: oral squamous cell carcinoma $\left.{ }^{31-33}\right)$.

DNA copy number and loss of heterozygosity analyses

Copy number alterations (CNAs) were defined for each of the 15 vulvar SCCs using the ngCGH module and RankSegmentation statistical algorithm in NEXUS software v7.5 (Biodiscovery, Inc., El Segundo, CA, USA). ${ }^{34}$ Loss of heterozygosity (LOH) events were inferred using Sequenza. ${ }^{35}$ Identified CNAs and $\mathrm{LOH}$ events were manually curated based on the depth ratio and B allele frequency.

\section{RESULTS}

The catalog of somatic mutations

A total of 15 vulvar SCC genomes (6 HPV (+) and $9 \mathrm{HPV}$ $(-))$ and paired normal tissue genomes were analyzed in this study (Table 1). The coverage of the sequencing depth was a 
Table 2 Summary of comparison data between HPV (+) and HPV (-) vulvar cancer genomes

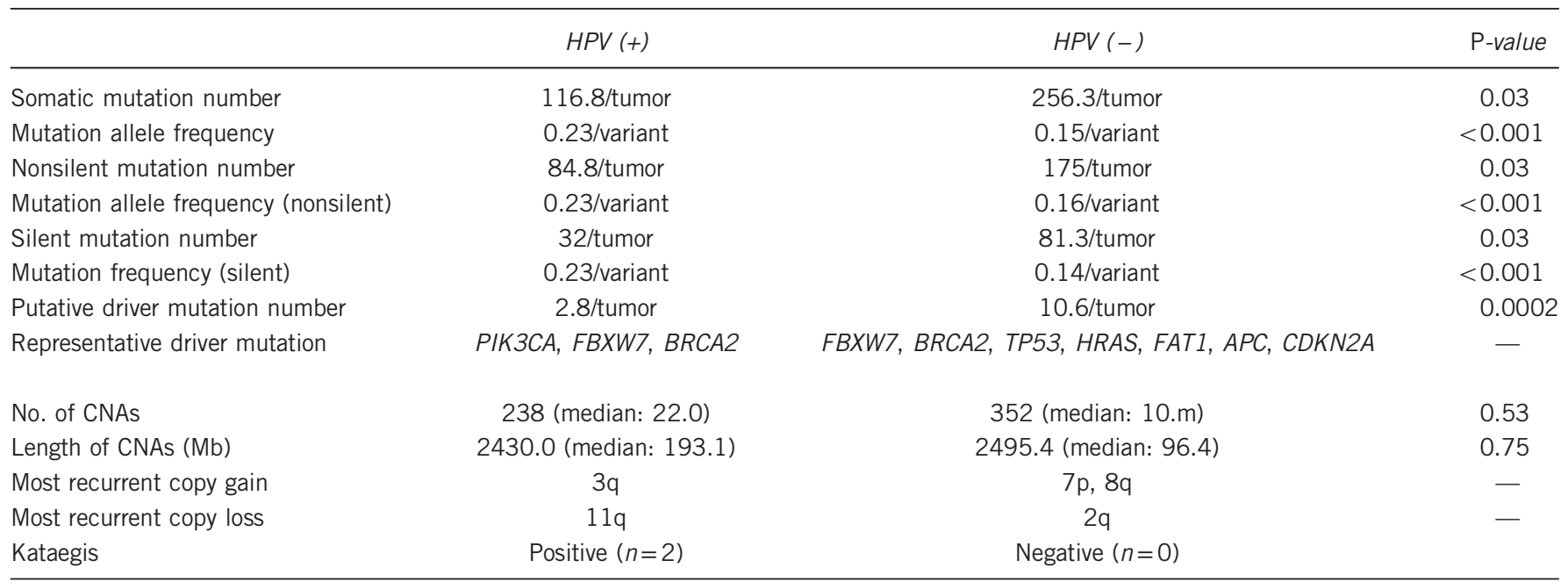

Abbreviation: CNAs, copy number alterations.

median of 141.8X (90.6-171.4X) for SCCs and 130.0X (60.6180.7X) for paired normal tissues (Supplementary Table S1). A total of 2084 nonsilent (27-297/per tumor; median of 130) and 924 silent mutations were identified in the vulvar SCC genomes with a mean rate of 5.6 somatic mutations per megabase (Figure 1a and b, Supplementary Table S2). These rates are higher than those of CESC (3.0 per megabase), ESCA (2.8), HNSC (3.3) and OSCC (2.6), but lower than those of CSCC (12.5) and LUSC (7.4). In terms of the sequence characteristics of point mutations, the $\mathrm{C}>\mathrm{T}$ substitution was the most common type $(31.0 \% ; 56.8 \%$ at $\mathrm{CpG}$ and $43.2 \%$ at non-CpG contexts) (Figure 1b). The frequencies of somatic nonsilent mutations in HPV ( - ) SCCs (average of 175 per tumor) were significantly higher than those of HPV (+) SCCs (average of 84.8 per tumor $)(P=0.03)$. The base substitutions were mainly $\mathrm{C}>\mathrm{T}$ or $\mathrm{C}>\mathrm{G}$ and did not differ between HPV $(+)$ and HPV ( - ) SCCs. Comparison data between HPV (+) and HPV (- ) SCCs are summarized in Table 2.

\section{Cancer-related genes}

Cancer-related genes were extracted from the Cancer Gene Census (http://cancer.sanger.ac.uk/census) ${ }^{36}$ and genes previously reported in vulvar SCCs. ${ }^{1,2,5,13-15}$ We identified a total of 56 cancer-related genes that contained not only known vulvar SCC mutations (TP53, CDKN2A, PIK3CA and HRAS) but also previously unknown vulvar SCC mutations, including $A P C, F B X W 7, B R C A 2, R B 1$ and FAT1 (Figure 2a). The putative driver mutations showed significantly higher mutation allele frequencies (mean MAFs; 0.19) than the other mutations (mean MAFs: 0.16, $P=0.02$ ). The PolyPhen-2 analysis ${ }^{26}$ revealed that the majority of the putative driver mutations (34 of 56, 61\%) were predicted to have damaging effects on protein function (Supplementary Table S3).

Recurrent nonsilent mutations ( $\geqslant 2$ cases) were found in 21 genes, including TP53 (5 cases), APC (3 cases), KMT2C (3 cases) and PIK3CA (2 cases) (Figure $2 \mathrm{a}$ ). The number of driver mutations in HPV (-) SCCs (average of 10.6 per tumor) was significantly higher than that of HPV (+) SCCs (average of 2.8 per tumor) $(P=0.0002)$. However, 4 of $6 \mathrm{HPV}(+)$ SCCs still harbored one of the known driver gene mutations (PIK3CA, $B R C A 2$ and FBXW7) (Table 2). There were more nonsynonymous mutations in HPV (-) SCCs (average of 6.8 per tumor) than HPV (+) SCCs (average of 1.5 per tumor) and TP53 was the most frequently mutated gene in the vulvar SCC genomes (Figure 2a).

Six truncating mutations were identified in known tumor suppressor genes (TSGs) FAT1, BRCA2, CASP8 and SMAD2. Of note, FAT1 harbored nonsilent mutations in 3 SCCs: VSCC15 with a nonsense mutation (p.W4175X), VSCC13 with 2 different nonsense mutations (p.S3268X and p.Q1455X) and VSCC9 with a missense mutation (p.D3749N). Three of six truncating mutations were identified in the FAT1 gene (p.W4175X, p.S3268X and p.Q1455X), which were located in known functional domains (cadherin domains and the intracytoplasmic domain) (Figure 2b).

\section{Mutation signatures}

We analyzed signatures of the somatic mutations detected in the vulvar SCCs using the NMF algorithm (Figure $3 \mathrm{a}$ and $\mathrm{b}$ and Supplementary Figure S1) and found that C:G>T:A transitions, which are frequently observed in mutations with oxidative DNA damage, ${ }^{37}$ were predominant in the vulvar SCCs. A decomposition of mutation spectra using the NMF algorithm revealed a pattern similar to signatures 1 and 2 in the COSMIC database (Figure 3c). Signature 1 (S1) is related to endogenous mutational processes present in most cancers and signature 2 (S2) is characterized by over-activity of the APOBEC cytidine deaminases. Three SCCs (VSCC3, 7 and 9) and another 3 (VSCC4, 14 and 15) SCCs dominantly exhibited S1 and S2, respectively. In terms of HPV status, there was no clear difference in the mutational signatures between HPV (+) and HPV ( - ) SCCs. However, the C $>\mathrm{T}$ transition, in the context of TpCpG trinucleotides, was more abundant in HPV (-) SCCs (average contribution of 0.17 per tumor) than in 
a

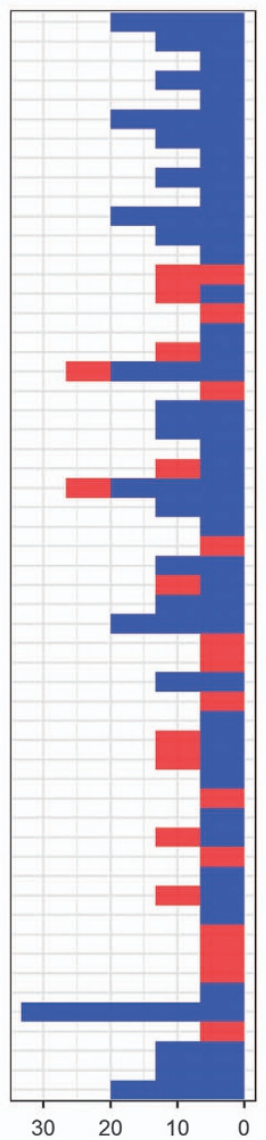

$\%$ Samples with Mutation
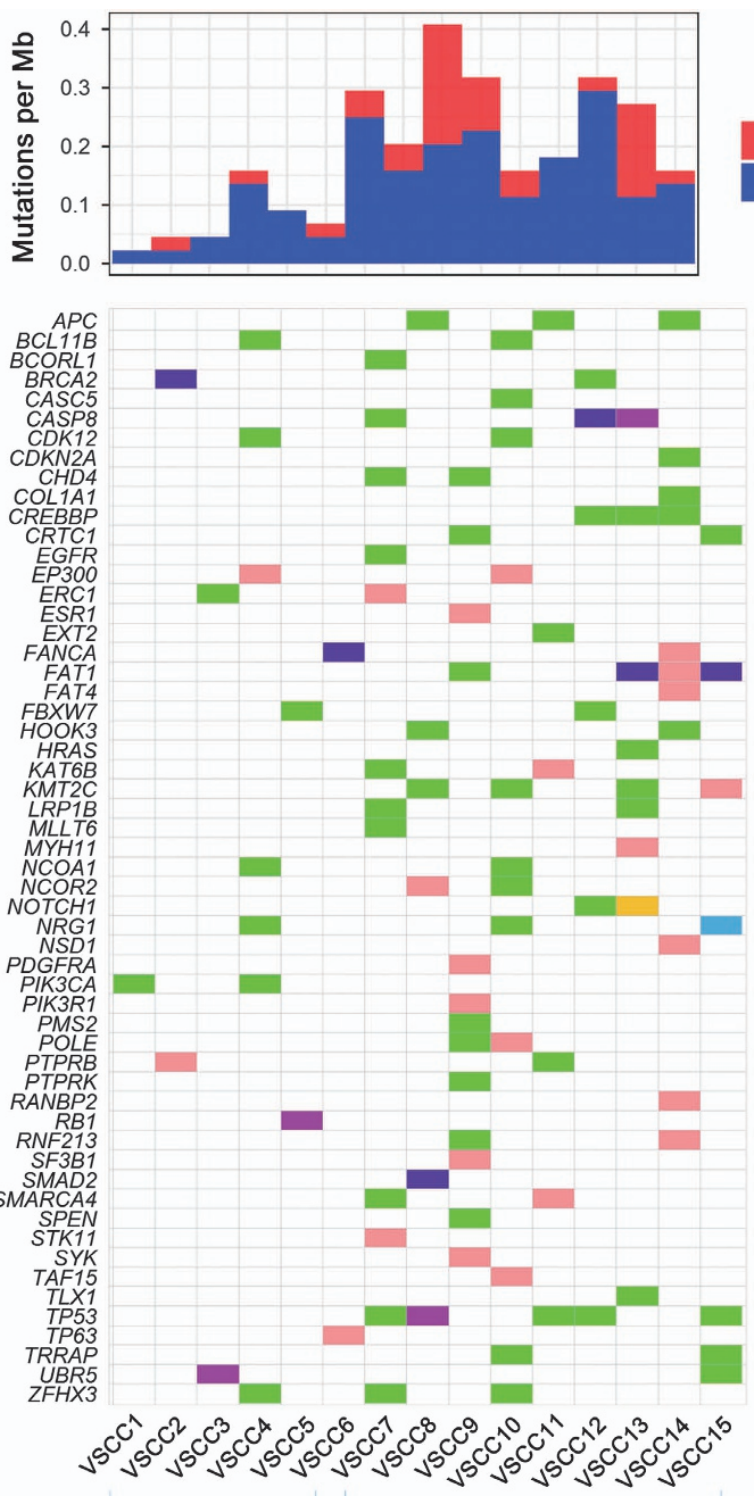

HPV (+)

HPV (-)
Synonymous

Non Synonymous

b

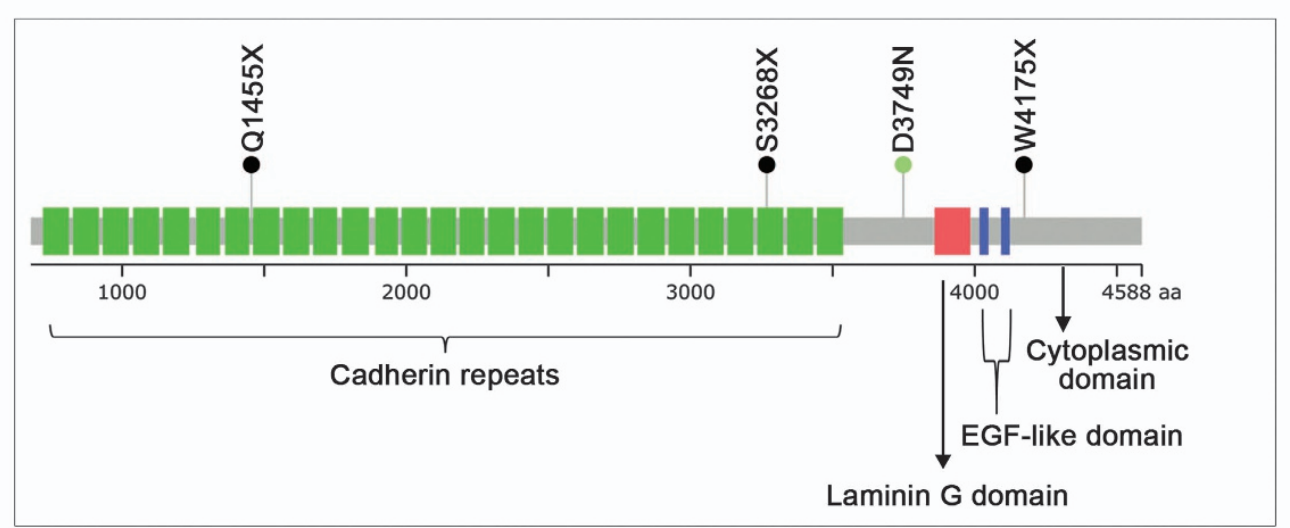

Figure 2 Putative driver mutations identified in vulvar SCCs. (a) Cancer-related genes listed in the Cancer Gene Census (http://cancer. sanger.ac.uk/census) identified in this study are shown for each sample. Fractions of each mutation in the SCCs are shown in the left plot. (b) Schematic representation of FAT1 protein domains and predicted locations of the mutations detected in vulvar SCCs. Black dot, truncating mutation; Green dot, missense mutation. 
a

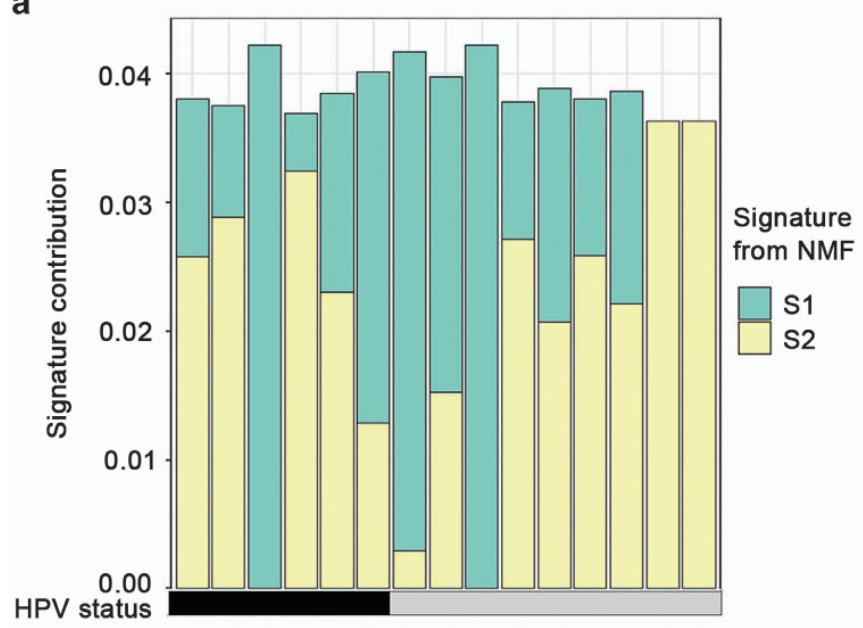

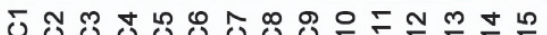

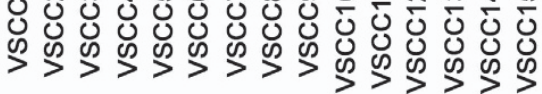

b

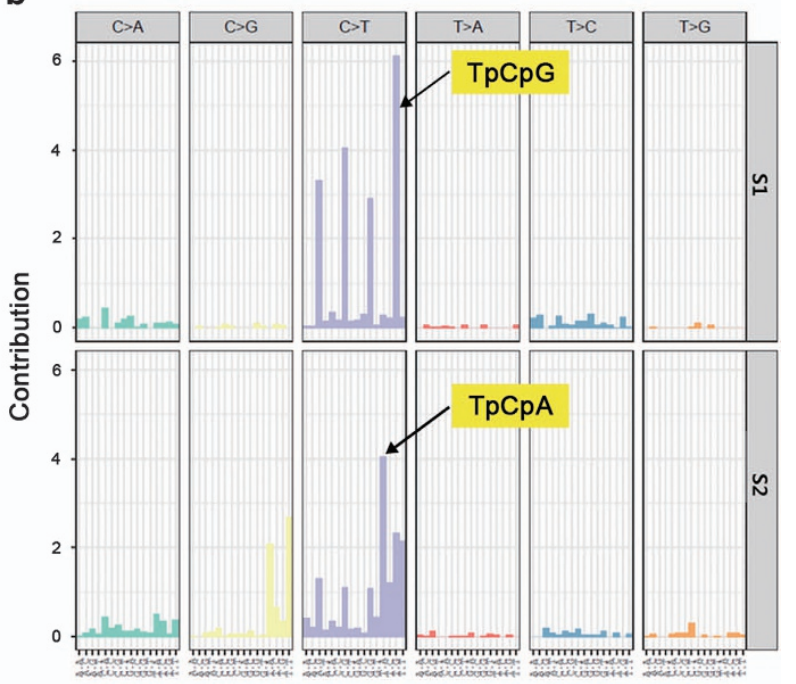

C

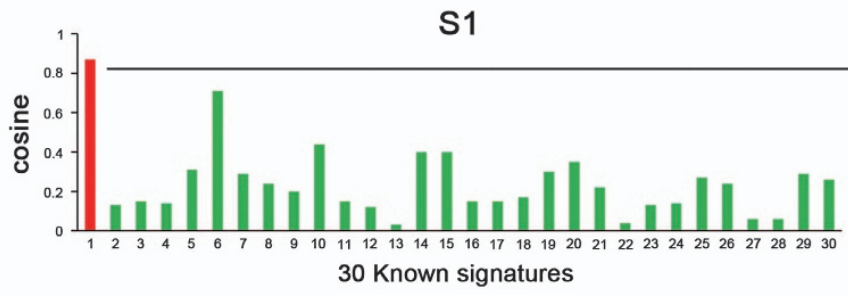

Spontaneous deamination of 5-methylcytosine (endogenous mutational process)

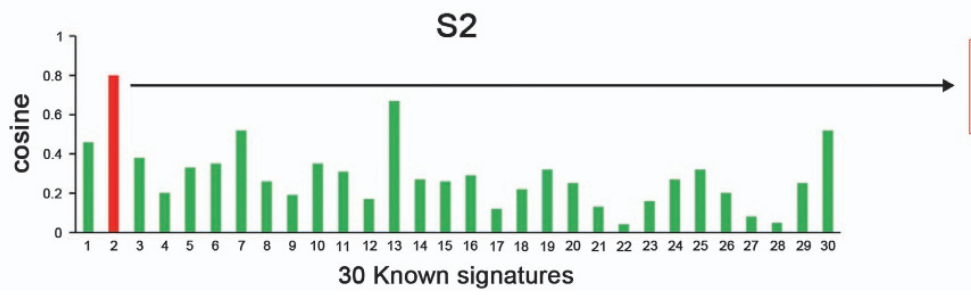

AID/APOBEC family of cytidine deaminases (most common in cervical and bladder cancers)

Figure 3 Mutation signatures of vulvar cancers. (a) The contribution of each signature to the filtered somatic mutation burden for each SCC. HPV status is shown in black (HPV $(+)$ ) and gray (HPV $(-)$ ). (b) Mutations within a trinucleotide context split by each of the two signatures generated from the NMF algorithm. (c) Each plot shows similarities between the mutational signatures from vulvar cancers and 30 known mutation signatures from the COSMIC database. Bars with high similarities are colored in red (cosine similarity $\geqslant 0.8$ ).

HPV (+) SCCs (average contribution of 0.14 per tumor). The contribution of the $\mathrm{C}>\mathrm{T}$ transition in the context of TpCpA trinucleotides was similar regardless of HPV status. When compared with other SCCs, cervical SCC showed the most similar mutation signature pattern (APOBEC family of cytidine deaminases) to the signature from the vulvar SCCs (Supplementary Figure S2).

\section{CNAs and $\mathrm{LOH}$}

By analyzing the WES data, a total of 590 CNAs (334 gains and 256 losses) were identified in 13 vulvar SCCs (Supplementary Table S4). Two vulvar SCCs (VSCC9 and 15) were not included due to low qualities, which were measured by data noise level (quality score of 0.157 and 0.099 for VSCC9 and 15, respectively). No significant difference in the number or length of CNAs was observed between HPV (+) and HPV (-) genomes $(P=0.53$ and 0.75 , respectively) (Table 2$)$. In the
HPV (+) SCCs, we found 15 recurrently altered CNA regions, where a gain on 3q25.33-q29 was the most recurrent event $(83 \%)$ (Table 3$)$. With regards to the HPV (-) SCCs, we found 18 recurrently altered CNA regions, where gains on $7 \mathrm{p}$ and $8 \mathrm{q}$ and a loss on $2 \mathrm{q}$ (all 57\%) were the most recurrent CNAs (Table 3). The CNA profiles were largely compatible with previously identified CNAs in vulvar SCCs (Table 3). ${ }^{38}$ In earlier studies, ${ }^{38}$ an $8 \mathrm{q}$ gain (86\%) and an $11 \mathrm{q}$ loss (29\%) were the most common CNAs in HPV (-) vulvar SCCs. In the present study, 13q, 18p-q, 20p, and 20q gains were newly discovered in the HPV (+) SCCs (Table 3). In the HPV (-) SCCs, gains at 7p, 10q, 14q and 21p- $\mathrm{q}$ and losses at $2 \mathrm{p}$ and Xp$\mathrm{q}$ were newly discovered (Table 3 ). Cancer-related genes such as FOXL2, PIK3CA, TP63, SOX2, TERT, BCL2, SMAD2 and GNAS in the regions of gains, as well as BIRC3 in the region of loss, were detected in the SCCs (Table 3, Figure $4 a$ and b). 
Table 3 Recurrently altered regions in vulvar SCCs by HPV status

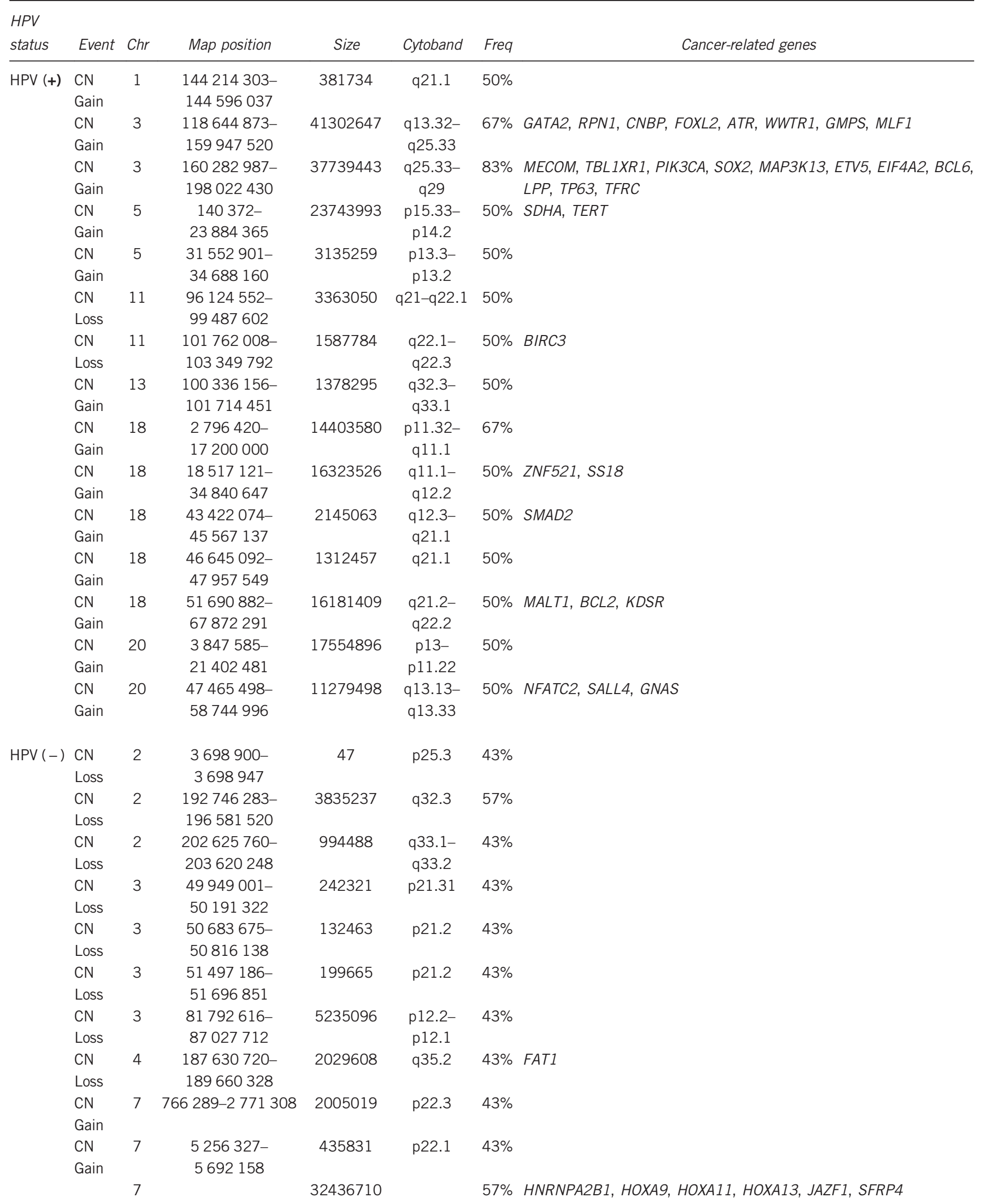


Table 3 (Continued)

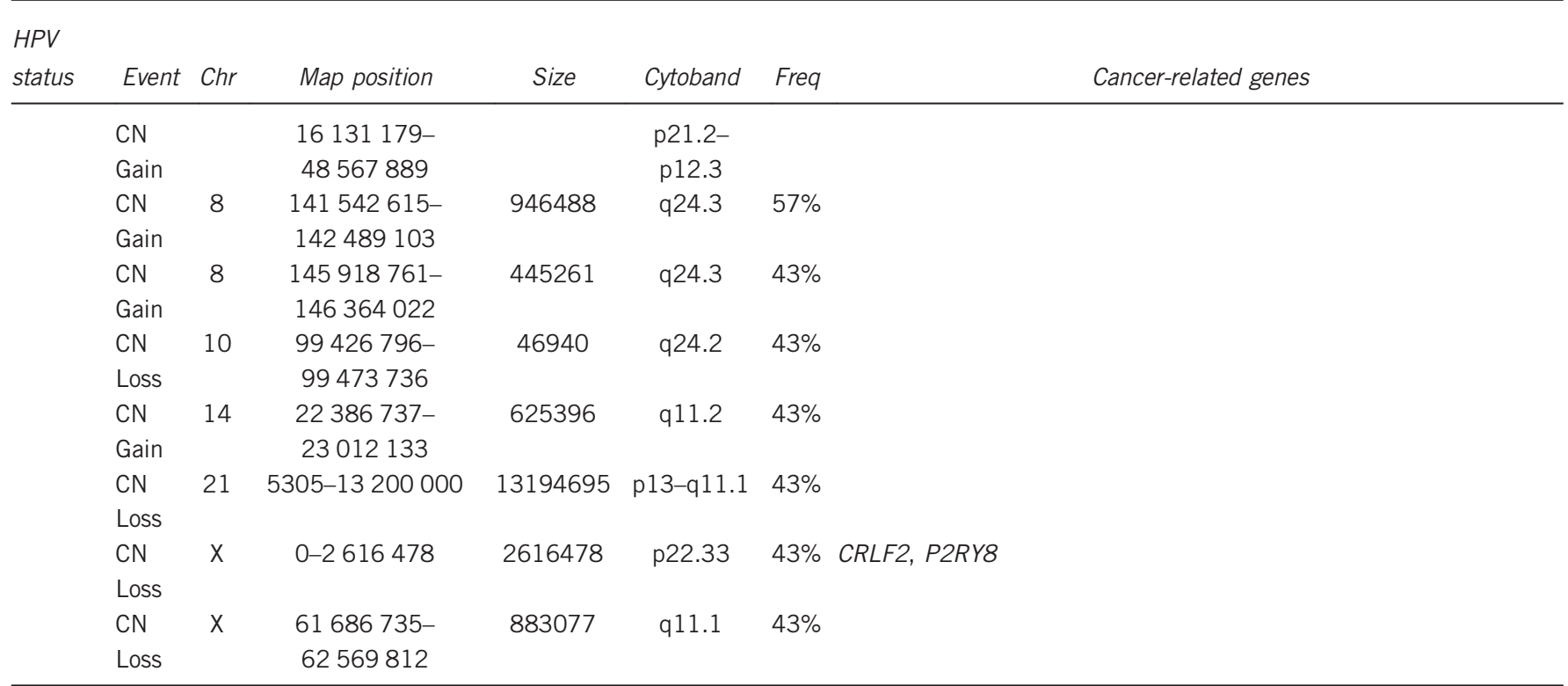

Abbreviations: CN gain, copy number gain; CN loss, copy number loss; Freq, frequency.

CNAs occurring in more than $40 \%$ of samples were defined as recurrently altered regions.

Cancer-related genes were extracted from the Cancer Gene Census (http://cancer.sanger.ac.uk/census) and genes previously reported in vulvar SCCs. ${ }^{1,2,5,13-15}$

Importantly, 4 of $6 \mathrm{HPV}(+)$ cases (VSCC1-4) showed copy gains at the PIK3CA locus (3q26.32). Two of them (VSCC1 and 4) harbored a hotspot missense mutation (p.E545K) (Figure 4c). The MAF of the missense mutation at the PIK3CA copy gain locus was $49 \%$ and the mean MAF in the copynumber neutral region was $24 \%$ in VSCC4. Similarly, in VSCC1, the MAF of the mutation in the PIK3CA copy gain locus was $21 \%$ and the mean MAF in the copy-number neutral region was $17 \%$. Although further experimental validation is required, these data suggest that these two events may have occurred in the same allele. Two of 7 HPV (-) SCCs also exhibited copy gains at the PIK3CA locus.

Next, we assessed the B-allele profiles of the 13 vulvar SCCs and identified 6 copy-neutral LOH events (2q33.1-q37.3, 3q11.2-q29, 7p22.3-p11.2, 7q22.1-q36.3, 9q22.2-q34.3 and 17p13.3-p11.2) in $4(31 \%)$ cases (1 HPV (+) and $3 \mathrm{HPV}$ $(-))$ where important cancer-related genes are located, including TP53, PIK3CA, NOTCH1, CASP8, EGFR, BRAF, FOXL2 and TP63 (Figure 5).

\section{Localized hypermutation}

Two vulvar SCCs with HPV (+) had a recurrent region of kataegis (a focus of localized substitution hypermutations) at chromosome 16p12.3-q22.2 (chr16:19566 736-72 137834 ) (Figure 6). The mutations in kataegis are usually characterized by clusters of $\mathrm{C}>\mathrm{T}$ and/or $\mathrm{C}>\mathrm{G}$ in the text of $\mathrm{TpC}$ dinucleotides. ${ }^{39}$ In agreement, we observed that most mutations in the kataegis region involved Tp$\underline{\mathrm{C}} \mathrm{N}$ mutations, with an inter-mutational distance of no more than $10 \mathrm{~kb}$ (Figure 6). ${ }^{27}$ Of note, the kataegis region in our study colocalized with the copy gain of the DHX38 gene, which is involved in RNA splicing.

\section{DISCUSSION}

Although the mutational landscape of cervical SCCs is already known, ${ }^{9}$ that of vulvar SCCs is unknown. The aim of this study was two-fold. First, we attempted to disclose somatic mutations and CNAs of vulvar SCCs genome-wide. Second, we attempted to disclose genomic differences between HPV (+) and HPV (-) vulvar SCCs. We found that the vulvar SCCs harbored not only known mutations in driver genes such as TP53, PIK3CA and HRAS but also novel mutations in cancer-related genes such as $A P C, F B X W 7, B R C A 2, R B 1$ and FAT1. We also found mounting evidence that HPV (+) and HPV (-) SCCs may differ with respect to genetic alterations, some of which were previously unknown. For example, the TP53 mutation was known as a sole driver mutation in HPV (+) vulvar SCCs ${ }^{13}$ but other driver gene mutations (PIK3CA, BRCA2 and FBXW7) were detected in the HPV (+) SCCs in the present study.

Most recurrent CNAs of HPV (+) SCCs in the present study were largely in agreement with those described in earlier studies, while those of HPV $(-)$ were not, except for the gain on $8 \mathrm{q} .{ }^{15}$ Of note, the gain on $3 \mathrm{q}$ was the most recurrent $\mathrm{CNA}$ in the HPV (+) vulvar SCCs, while the gains on $7 \mathrm{p}$ and $8 \mathrm{q}$ and the loss on $2 \mathrm{q}$ were the most recurrent in HPV $(-)$ cases. Unlike somatic mutations that exhibited higher frequency in HPV (-) SCCs than in HPV (+) SCCs, the CNA frequencies between HPV (-) and HPV (+) vulvar SCC genomes did not differ. Together, our data suggest that a $3 \mathrm{q}$ gain where PIK3CA is located plays a role in vulvar SCC development irrespective of the HPV status and that the other CNAs could be related to HPV status. In the 5 SCCs (2 HPV (+) and $3 \mathrm{HPV}(-))$, we found a copy-neutral $\mathrm{LOH}$, also called uniparental disomy (UPD), in which there are two copies of a chromosome from one parent and no copies from the other parent due to errors 
a

Whole chromosome

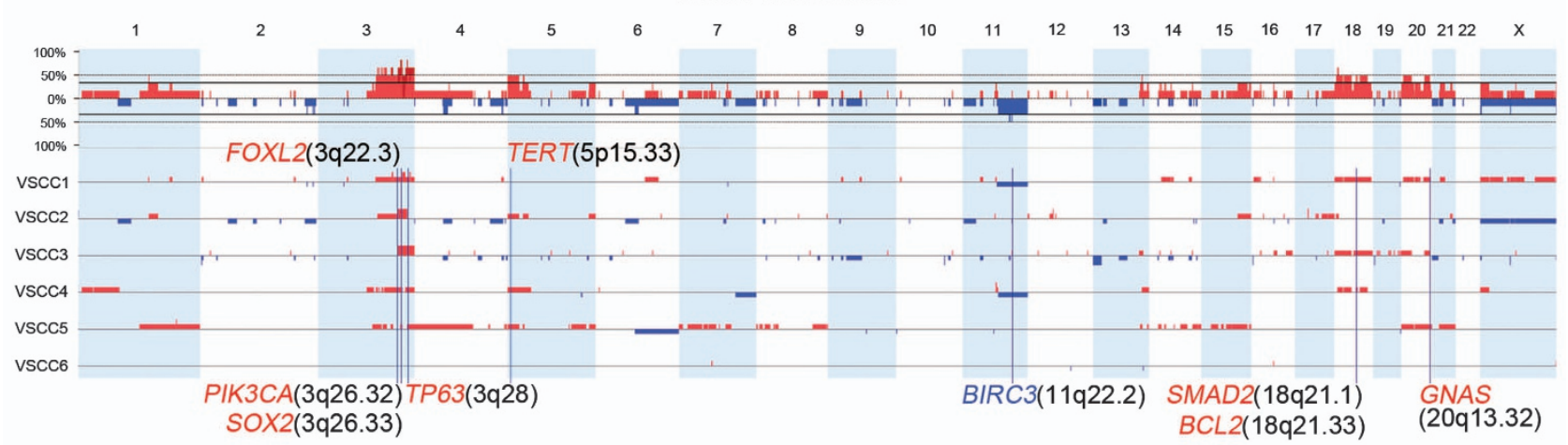

b

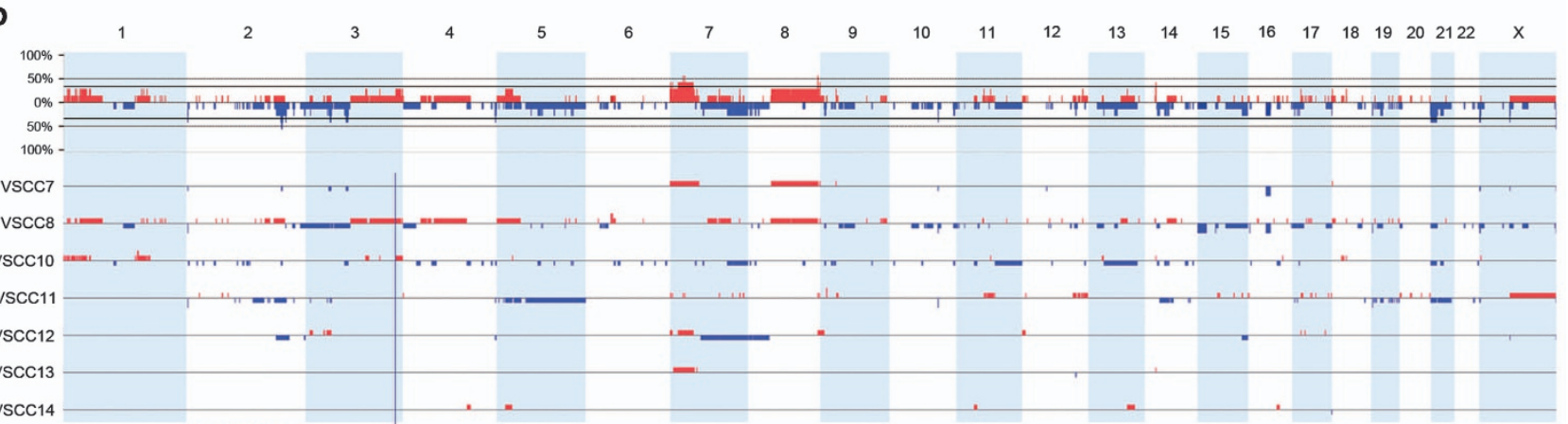

PIK3CA(3q26.32)

C

Chromosome 3
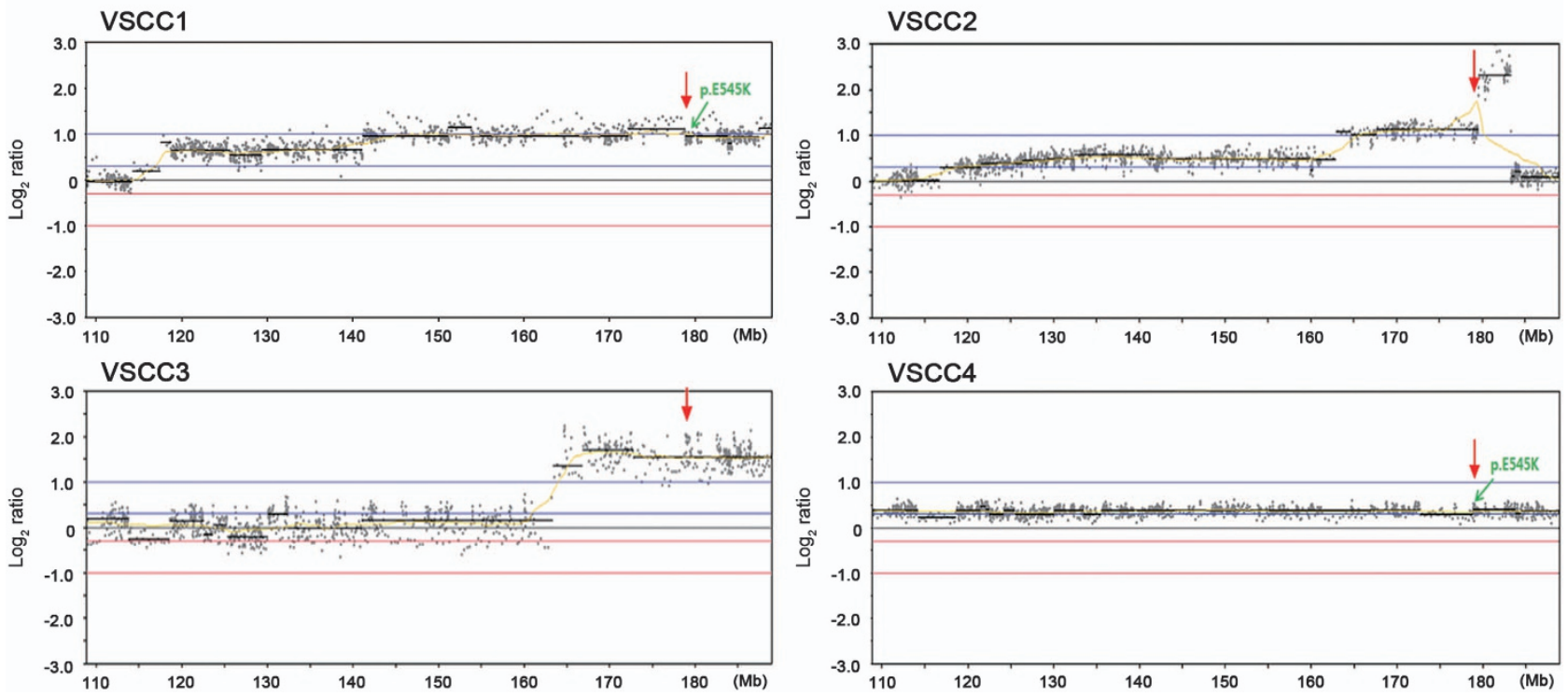

Figure 4 Copy number alterations identified in HPV (+) vulvar SCCs. (a) Genome-wide frequency of copy number alterations in HPV (+) SCCs. Gain (positive axis, red). Loss (negative axis, blue). (b) Genome-wide frequency of copy number alterations in HPV (-) SCCs. (c) Focal amplification at the PIK3CA gene locus (3q26.32, red arrows) identified in four HPV (+) SCCs. Green arrows indicate PIK3CA missense mutations (p.E545K) in VSCC1 and 4.

in meiosis I or meiosis II. $^{40}$ The copy-neutral LOH may contribute to the development of tumors that are homozygous for preexisting mutations. ${ }^{41}$ The copy-neutral $\mathrm{LOH}$ areas in the vulvar SCCs identified in this study harbored tumor suppressor genes (TP53 and CASP8) and an oncogene (PIK3CA). We are the first to report this copy-neutral LOH in vulvar SCCs; however, its distribution as well as its biological and clinical significance in vulvar SCCs remains to be clarified.

Diverse endogenous and exogenous mutational processes leave distinct patterns of mutational signatures embedded within cancer genomes. ${ }^{42}$ Signature 1 , initiated by the deamination of 5-methyl-cytosine, is found in the majority of human 
a

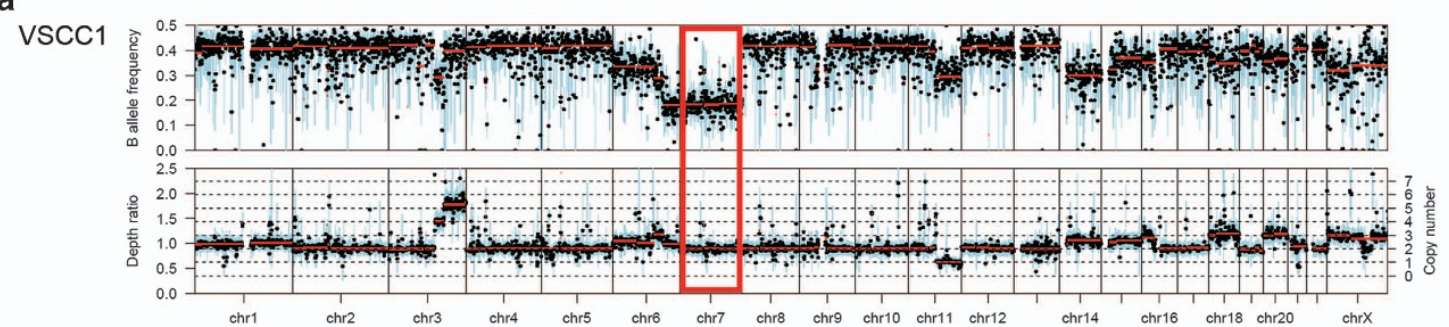

VScc7

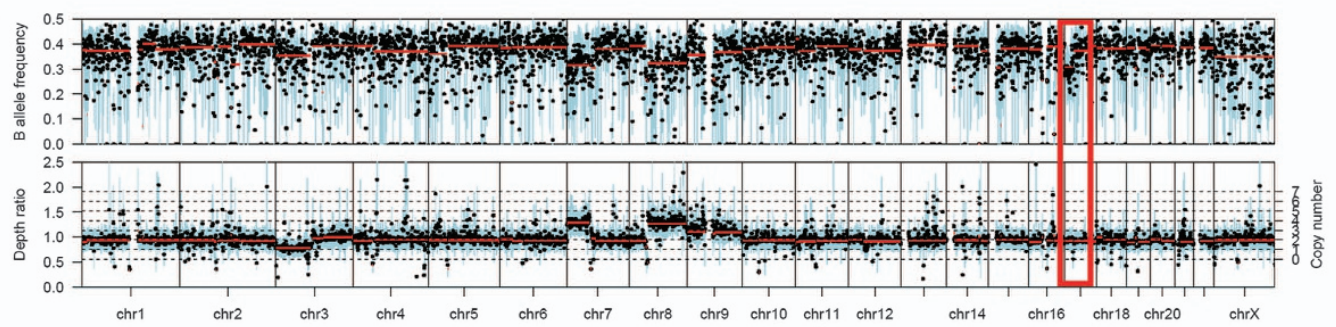

VScc12

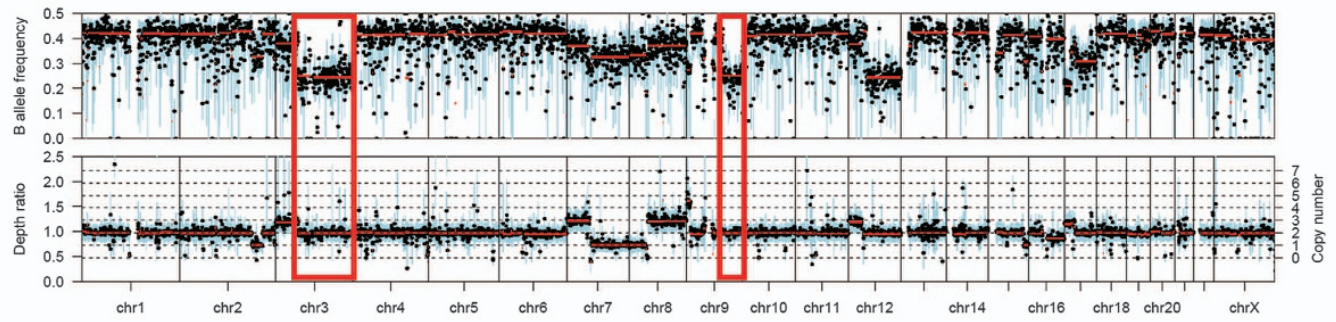

VScc13

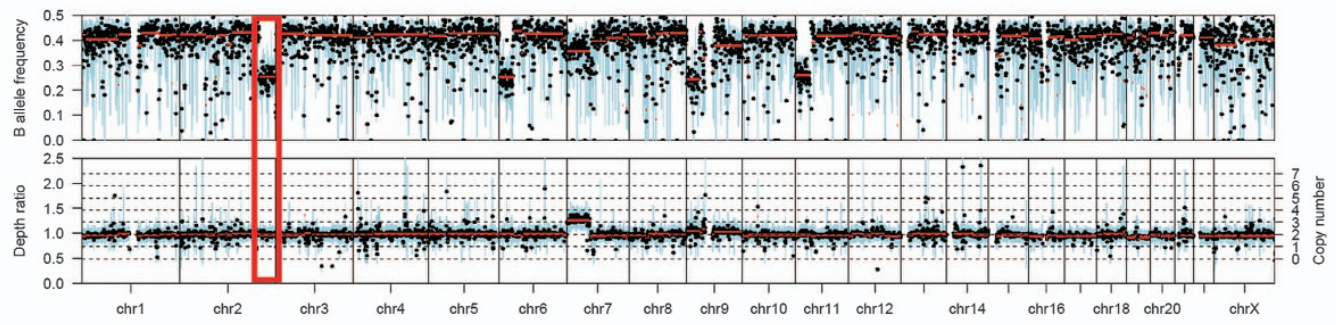

b

\begin{tabular}{|c|c|c|c|c|}
\hline Sample & HPV status & Genomic position* & Event & Cancer-related genes** \\
\hline VSCC1 & HPV (+) & $\begin{array}{l}\text { chr7:331086-57997338, } \\
\text { chr7:100610026-159026164 }\end{array}$ & Copy-neutral LOH & $E G F R, B R A F$ \\
\hline VSCC7 & HPV (-) & chr17:6115-21906705 & Copy-neutral LOH & TP53 \\
\hline VSCC12 & HPV (-) & $\begin{array}{l}\text { chr3:97365038-197952910, } \\
\text { chr9:93205777-141071475 }\end{array}$ & Copy-neutral LOH & $\begin{array}{l}\text { PIK3CA, FOXL2, TP63, } \\
\text { NOTCH1 }\end{array}$ \\
\hline VSCC13 & HPV (-) & chr2:201719211-243152744 & Copy-neutral LOH & CASPB \\
\hline
\end{tabular}

* UCSC GRCh37/hg19

** Cancer-related genes are extracted from Cancer Gene Census (http://cancer.sanger.ac.uk/census) and genes previously reported in vulvar SCCs.

Figure 5 Copy-neutral loss of heterozygosity ( $\mathrm{LOH}$ ) events identified in vulvar cancers. (a) Copy-neutral LOH events at cancer-related gene loci are highlighted with red square boxes. The upper bar represents the B allele frequency (0.0-0.5) and the lower bar represents the depth ratio (0.0-2.5). (b) List of copy-neutral LOH events identified in vulvar cancers. Four vulvar SCCs with copy-neutral LOH at cancerrelated gene loci.

cancers $^{43}$ and has a probable association with aging. ${ }^{42}$ Signature 2 is the second most common signature related to the APOBEC family of cytidine deaminases coupled with DNA replication and repair machineries. ${ }^{39,44}$ The co-occurrence of these two signatures is found in SCCs from the cervix, head/ neck and esophagus. ${ }^{42}$ The vulvar SCCs examined in this study had both signatures 1 and 2 without any other distinct signatures. These data suggest that vulvar SCCs may not harbor 
a
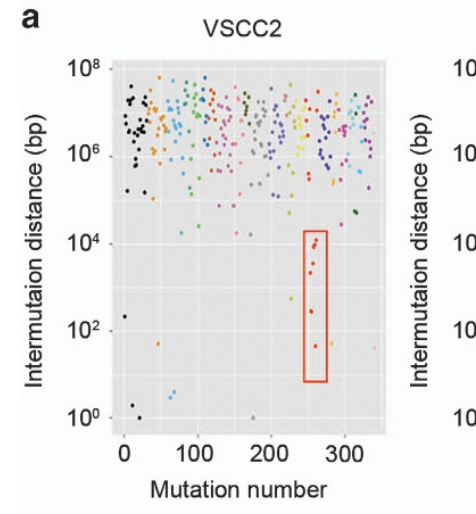

vscc2

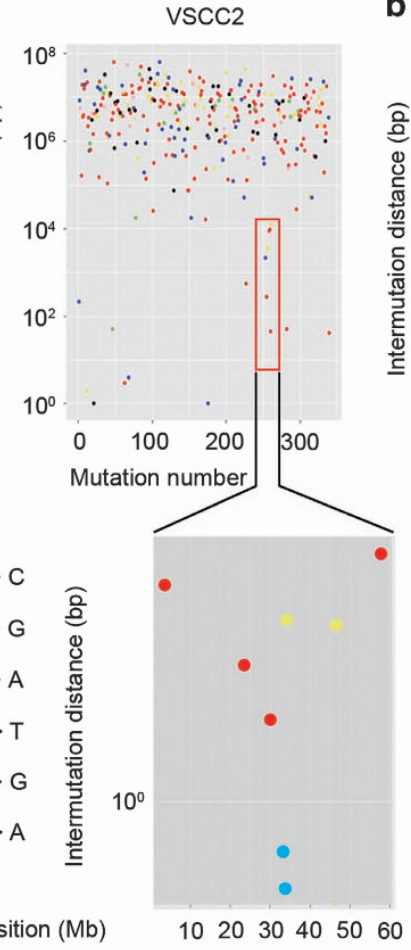

Chromosome 16 b

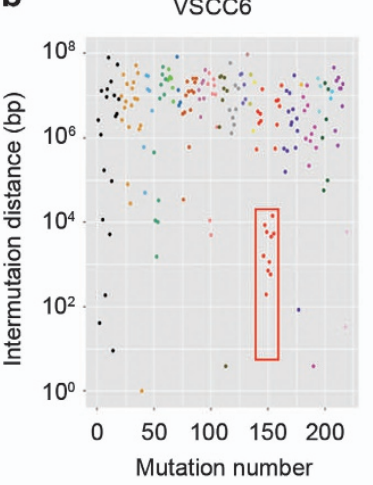

vscc6

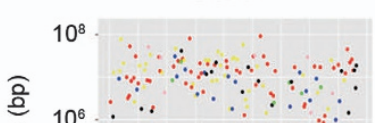

1

$10^{4}$

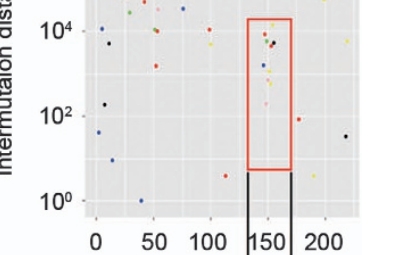

Mutation number

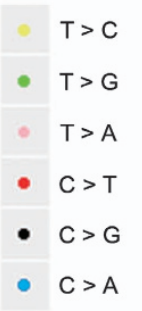

Position (Mb)

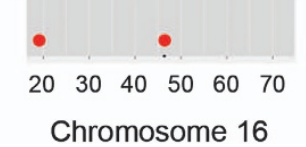

Figure 6 Kataegis identified in vulvar cancers. Regional hypermutation plots in two cancers. Upper panels of a and $\mathbf{b}$ : the left plot represents an individual sample in which each dot represents a single somatic mutation colored on a chromosomal basis. The right plot represents the same data colored by mutation type. Mutations are ordered consecutively on the $x$ axis from the first mutation in the short arm of chromosome 1 to the last mutation in the long arm of chromosome X. Kataegis at chromosome 16 is highlighted by a red square box. Lower panels of $\mathbf{a}$ and $\mathbf{b}$ : kataegis loci are shown on chromosome 16.

any other distinct mutational etiologies such as alcohol, tobacco and ultraviolet exposure besides HPV and aging.

In two HPV (+) SCCs, we found recurrent kataegis, a process related to the activation of APOBEC enzymes, at 16p12.3-16q22.2 on the basis of substitution type and sequence context of cytosine mutations. ${ }^{39,42}$ Currently, kataegis is not defined by a statistics-based analysis; therefore, although our observation of a kataegis-enriched mutation is in agreement with a previous report, ${ }^{39}$ a proper statistical method for such purposes should be developed. APOBEC activity is induced in HPV-infected cells and is highly associated with HPV (+) head/ neck cancer. ${ }^{45}$ The 16q22.2 locus (with the kataegis) encompasses the DHX38 gene that encodes an RNA helicase and is involved in RNA splicing. ${ }^{46}$ Whether the recurrent kataegis involving DHX38 is specific for the tumorigenesis of vulvar SCCs remains to be studied in a larger cohort.

The most common mutation identified in this study was the TP53 mutation (5/15), consistent with previous studies. ${ }^{5}$ The TP53 mutation in one case (VSCC8) was accompanied by a TP53 copy loss, which would result in the complete inactivation of TP53. Six vulvar SCCs showed PIK3CA copy gains. Of them, 2 cases (VSCC1 and 4) harbored concurrent PIK3CA mutations, while the other 4 cases harbored only PIK3CA copy gains without any PIK3CA mutation. When combining the PIK3CA mutations with the copy gains, PIK3CA genetic alterations in vulvar SCCs reached $60 \%$, suggesting that it may be the major genetic alteration in vulvar SCCs. Further experimental validation of the concurrent PIK3CA alteration and its biological roles will be required. Additionally, $3 \mathrm{HPV}$ (-) SCCs harbored nonsense or missense mutations of the tumor suppressor gene FAT1, while the other $3 \mathrm{HPV}(-)$ SCCs harbored FAT1 copy losses. All of these events occurred in HPV ( - ) SCCs, suggesting that an alteration in FAT1 would be an HPV (- ) SCC-specific event, although the sample scale was not sufficient to conclude this hypothesis. Recurrent somatic mutations of FAT1 have been reported in multiple human cancers, including head/neck SCC, cervical adenocarcinoma and glioblastoma; ${ }^{47}$ they reported truncating mutations (p.R146M, p.W543X and p.E314X) in the cadherin and cytoplasmic domains of the FAT1 gene, the same locations as those reported in our study. ${ }^{47}$ In addition, FAT1 inactivation by somatic mutations activates Wnt signaling and promotes tumorigenesis. ${ }^{47}$ HPV (-) head/neck SCCs harbor frequent FAT1 mutations with loss of functions, which are significantly associated with better overall survival in HPV ( - ) patients but not in HPV (+) patients. ${ }^{48}$ Based on our data, the clinical significance of FAT1 inactivation in vulvar SCCs should be further examined in future studies.

Vulvar SCC has been divided into two different etiologic pathways (HPV-associated and HPV-non-associated). 
However, the genetic differences between these two have not been studied by genome-wide analyses. Based on the present study, the following conclusions were made: (i) the overall loads for somatic mutations (total mutations, nonsilent mutations and driver mutations) are higher in HPV ( - ) SCCs than in HPV (+) SCCs; (ii) the overall loads for CNAs (length and number) are not different; and (iii) the profiles of driver mutations and CNAs overlap but are not identical (Table 2). Several HPV-induced genes such as E6 and E7 act as oncogenes that promote tumor growth and malignant transformation, which could render HPV-infected cells less independent of additional somatic mutations for cancer development than HPV-uninfected cells. ${ }^{49,50}$ Because there was no difference in the overall CNA loads, it can be hypothesized that HPV status may not have substantial effects during SCC development. The overlapping but different profiles of driver mutations and CNAs between HPV (+) and HPV (-) SCCs suggest that vulvar SCC development may require genetic alterations specific to either HPV-dependent or HPV-independent lesions in addition to common genetic alterations of HPV-dependent and HPV-independent SCCs.

In summary, we report the global genomic alteration profiles of vulvar SCCs to provide a better understanding of both the HPV-dependent and HPV-independent pathogenesis of vulvar SCCs. The genomic features of vulvar SCCs identified in the current study aid in a better understanding of the etiology of vulvar SCCs.

\section{CONFLICT OF INTEREST}

The authors declare no conflict of interest.

\section{ACKNOWLEDGEMENTS}

This study was supported by a grant from the National Research Foundation of Korea (2012R1A5A2047939) and by a grant from the Korea Healthcare Technology R\&D Project (HI14C3417 and HI13C2148).

\section{PUBLISHER'S NOTE}

Springer Nature remains neutral with regard to jurisdictional claims in published maps and institutional affiliations.

1 del Pino M, Rodriguez-Carunchio L, Ordi J. Pathways of vulvar intraepithelial neoplasia and squamous cell carcinoma. Histopathology 2013; 62: 161-175.

2 Clancy AA, Spaans JN, Weberpals JI. The forgotten woman's cancer: vulvar squamous cell carcinoma (VSCC) and a targeted approach to therapy. Ann Oncol 2016; 27: 1696-1705.

3 zur Hausen H. Papillomaviruses and cancer: from basic studies to clinical application. Nat Rev Cancer 2002; 2: 342-350.

4 Woodman CBJ, Collins SI, Young LS. The natural history of cervical HPV infection: unresolved issues. Nat Rev Cancer 2007; 7: 11-22.

5 Trietsch MD, Nooij LS, Gaarenstroom KN, van Poelgeest MI. Genetic and epigenetic changes in vulvar squamous cell carcinoma and its precursor lesions: a review of the current literature. Gynecol Oncol 2015; 136: 143-157.

6 Hoang LN, Park KJ, Soslow RA, Murali R. Squamous precursor lesions of the vulva: current classification and diagnostic challenges. Pathology 2016; 48: 291-302.
7 Cormio G, Loizzi V, Carriero C, Cazzolla A, Putignano G, Selvaggi L. Groin recurrence in carcinoma of the vulva: management and outcome. Eur J Cancer Care 2010; 19: 302-307.

8 Network TCGA, Comprehensive molecular characterization of human colon and rectal cancer. Nature 2012; 487: 330-337.

9 Ojesina Al, Lichtenstein L, Freeman SS, Pedamallu CS, Imaz-Rosshandler I, Pugh TJ et al. Landscape of genomic alterations in cervical carcinomas. Nature 2014; 506: 371-375.

10 Gao Y-B, Chen Z-L, Li J-G, Hu X-D, Shi X-J, Sun Z-M et al. Genetic landscape of esophageal squamous cell carcinoma. Nat Genet 2014; 46: 1097-1102.

11 Pickering CR, Zhou JH, Lee JJ, Drummond JA, Peng SA, Saade RE et al. Mutational landscape of aggressive cutaneous squamous cell carcinoma. Clin Cancer Res Off J Am Assoc Cancer Res 2014; 20: 6582-6592.

12 Hedberg ML, Goh G, Chiosea SI, Bauman JE, Freilino ML, Zeng Y et al. Genetic landscape of metastatic and recurrent head and neck squamous cell carcinoma. J Clin Invest 126: 169-180.

13 Trietsch MD, Spaans VM, ter Haar NT, Osse EM, Peters AAW, Gaarenstroom KN et al. CDKN2A(p16) and HRAS are frequently mutated in vulvar squamous cell carcinoma. Gynecol Oncol 2014; 135: 149-155.

14 Kunjoonju JP, Raitanen M, Grénman S, Tiwari N, Worsham MJ. Identification of individual genes altered in squamous cell carcinoma of the vulva. Genes Chromosomes Cancer 2005; 44: 185-193.

15 Zhang L, Wu J, Ling MT, Zhao L, Zhao K-N. The role of the PI3K/Akt/mTOR signalling pathway in human cancers induced by infection with human papillomaviruses. Mol Cancer 2015; 14: 87.

16 Li YY, Hanna GJ, Laga AC, Haddad RI, Lorch JH, Hammerman PS. Genomic analysis of metastatic cutaneous squamous cell carcinoma. Clin Cancer Res 2015; 21: 1447-1456.

17 Network TCGA. Comprehensive genomic characterization of head and neck squamous cell carcinomas. Nature 2015; 517: 576-582.

18 Jung S-H, Choi YJ, Kim MS, Baek I-P, Lee SH, Lee AW et al. Progression of naive intraepithelial neoplasia genome to aggressive squamous cell carcinoma genome of uterine cervix. Oncotarget 2015; 6: 4385-4393.

19 Feber A, Worth DC, Chakravarthy A, de Winter P, Shah K, Arya M et al. CSN1 somatic mutations in penile squamous cell carcinoma. Cancer Res 2016; 76: 4720-4727.

20 Kim T-M, Jung S-H, Kim MS, Baek I-P, Park S-W, Lee SH et al. The mutational burdens and evolutionary ages of early gastric cancers are comparable to those of advanced gastric cancers. J Pathol 2014; 234: 365-374.

$21 \mathrm{Li} \mathrm{H}$, Durbin R. Fast and accurate short read alignment with BurrowsWheeler transform. Bioinformatics 2009; 25: 1754-1760.

22 DePristo MA, Banks E, Poplin R, Garimella KV, Maguire JR, Hartl C et al. A framework for variation discovery and genotyping using next-generation DNA sequencing data. Nat Genet 2011; 43: 491-498.

$23 \mathrm{Li} \mathrm{H}$, Handsaker B, Wysoker A, Fennell T, Ruan J, Homer N et al. The Sequence Alignment/Map format and SAMtools. Bioinformatics 2009; 25 : 2078-2079.

24 Cibulskis K, Lawrence MS, Carter SL, Sivachenko A, Jaffe D, Sougnez C et al. Sensitive detection of somatic point mutations in impure and heterogeneous cancer samples. Nat Biotechnol 2013; 31: 213-219.

25 Wang K, Li M, Hakonarson H. ANNOVAR: functional annotation of genetic variants from high-throughput sequencing data. Nucleic Acids Res 2010; 38: e164-e164.

26 Adzhubei IA, Schmidt S, Peshkin L, Ramensky VE, Gerasimova A, Bork P et al. A method and server for predicting damaging missense mutations. Nat Methods 2010; 7: 248-249.

27 Roberts SA, Sterling J, Thompson C, Harris S, Mav D, Shah R et al. Clustered mutations in yeast and in human cancers can arise from damaged long single-strand DNA regions. Mol Cell 2012; 46: 424-435.

28 Gehring JS, Fischer B, Lawrence M, Huber W. SomaticSignatures: inferring mutational signatures from single-nucleotide variants. Bioinformatics 2015; 31: 3673-3675.

29 Forbes SA, Beare D, Gunasekaran P, Leung K, Bindal N, Boutselakis H et al. COSMIC: exploring the world's knowledge of somatic mutations in human cancer. Nucleic Acids Res 2015; 43: D805-D811.

30 Network TCGA, Comprehensive molecular portraits of human breast tumours. Nature 2012; 490: 61-70.

31 Pickering CR, Zhang J, Yoo SY, Bengtsson L, Moorthy S, Neskey DM et al. Integrative genomic characterization of oral squamous cell carcinoma identifies frequent somatic drivers. Cancer Discov 2013; 3: 770-781.

32 Hayes TF, Benaich N, Goldie SJ, Sipilä K, Ames-Draycott A, Cai W et al. Integrative genomic and functional analysis of human oral squamous cell carcinoma cell lines reveals synergistic effects of FAT1 and CASP8 inactivation. Cancer Lett 2016; 383: 106-114. 
33 India Project Team of the International Cancer Genome Consortium. Mutational landscape of gingivo-buccal oral squamous cell carcinoma reveals new recurrently-mutated genes and molecular subgroups. Nat Commun 2013; 4: 2873.

34 Jung S-H, Shin S, Kim MS, Baek I-P, Lee JY, Lee SH et al. Genetic progression of high grade prostatic intraepithelial neoplasia to prostate cancer. Eur Urol 2016; 69: 823-830.

35 Favero F, Joshi T, Marquard AM, Birkbak NJ, Krzystanek M, Li Q et al. Sequenza: allele-specific copy number and mutation profiles from tumor sequencing data. Ann Oncol 2015; 26: 64-70.

36 Futreal PA, Coin L, Marshall M, Down T, Hubbard T, Wooster R et al. A census of human cancer genes. Nat Rev Cancer 2004; 4: 177-183.

37 Dizdaroglu M. Oxidatively induced DNA damage: mechanisms, repair and disease. Cancer Lett 2012; 327: 26-47.

38 Thomas LK, Bermejo JL, Vinokurova S, Jensen K, Bierkens M, Steenbergen $\mathrm{R}$ et al. Chromosomal gains and losses in human papillomavirus-associated neoplasia of the lower genital tract - a systematic review and meta-analysis. Eur J Cancer 2014; 50: 85-98.

39 Nik-Zainal S, Alexandrov LB, Wedge DC, Van Loo P, Greenman CD, Raine K et al. Mutational processes molding the genomes of 21 breast cancers. Cell 2012; 149: 979-993.

40 Tuna M, Knuutila S, Mills GB. Uniparental disomy in cancer. Trends $\mathrm{Mo}$ Med 2009; 15: 120-128.

41 Dunbar AJ, Gondek LP, O'Keefe CL, Makishima H, Rataul MS, Szpurka H et al. 250K SNP array karyotyping identifies acquired uniparental disomy and homozygous mutations, including novel missense substitutions of c-Cbl, in myeloid malignancies. Cancer Res 2008; 68: 10349-10357.

42 Alexandrov LB, Nik-Zainal S, Wedge DC, Aparicio SAJR, Behjati S, Biankin $\mathrm{AV}$ et al. Signatures of mutational processes in human cancer. Nature 2013; 500: 415-421.

43 Pfeifer GP. Mutagenesis at methylated CpG sequences. Curr Top Microbiol Immunol 2006; 301: 259-281.

44 Noia JMD, Neuberger MS. Molecular mechanisms of antibody somatic hypermutation. Annu Rev Biochem 2007; 76: 1-22.
45 Henderson S, Chakravarthy A, Su X, Boshoff C, Fenton TR. APOBECmediated cytosine deamination links PIK3CA helical domain mutations to human papillomavirus-driven tumor development. Cell Rep 2014; 7: 1833-1841.

46 Han S, Park J, Lee D-H. Protein DHX38 is a novel inhibitor of protein phosphatase 4. Anim Cells Syst 2015; 19: 236-244.

47 Morris LGT, Kaufman AM, Gong Y, Ramaswami D, Walsh LA, Turcan S et al. Recurrent somatic mutation of FAT1 in multiple human cancers leads to aberrant Wnt activation. Nat Genet 2013; 45: 253-261.

$48 \mathrm{Kim} \mathrm{KT}$, Kim B-S, Kim JH. Association between FAT1 mutation and overall survival in patients with human papillomavirus-negative head and neck squamous cell carcinoma. Head Neck 2016; 38: E2021-E2029.

49 Yim E-K, Park J-S. The role of HPV E6 and E7 oncoproteins in HPVassociated cervical carcinogenesis. Cancer Res Treat Off J Korean Cancer Assoc 2005; 37: 319-324.

50 Tomaić V. Functional roles of E6 and E7 oncoproteins in HPV-induced malignancies at diverse anatomical sites. Cancers 2016; 8: pii: E95.

(c) (1) $(\Theta$ This work is licensed under a Creative Commons Attribution-NonCommercial-NoDerivs 4.0 International License. The images or other third party material in this article are included in the article's Creative Commons license, unless indicated otherwise in the credit line; if the material is not included under the Creative Commons license, users will need to obtain permission from the license holder to reproduce the material. To view a copy of this license, visit http://creativecommons.org/licenses/by-nc-nd/4.0/

(C) The Author(s) 2018

Supplementary Information accompanies the paper on Experimental \& Molecular Medicine website (http://www.nature.com/emm) 